\title{
Nouvelle méthode de détermination de la capacité d'absorption en terrains perméables
}

\section{A new method of determining the infiltration capacity of impermeable land}

\author{
A. BOUCHARDEAU \\ DIRECTEUR DE RECHERCHES \\ A L'OFFICE DF rA RECHERCHE SCIENTIFIQUE \\ ET TECHNIQUE OUTAE-MER
}

PAR

ET

Sur un bassin de faible superficie, la prévision de l'hydrogramme de crue correspondant à une averse donnée, se heurte à un probleme difficile: l'estimation de la capacité d'absorption.

En terrain perméable, les facteurs intervenant dans la détermination de cette grandenr sont multiples.

La détermination expérimentale de la hauteur limite de précipitations à partir de laquelle débute le ruissellement, ou pluie d'imbibition, permet de ramener ce problème à l'estimation de In capacité d'infiltration sur une surface hétérogène, ce qui est beaucoup plus facile.

L'emploi de la méthode Porcher donne une base solide pour l'extrapolation des capacités d'infiltration pour les très fortes intensités de précipitations.

Le calcul des crues exceptionnelles est rendu ainsi beaucoup moins aléatoire.

Cette méthode, valable pour les terrains de perméabilité moyenne ou forte, doit pouvoir rendre de grands services dans le cas d'épaisse couverture végétale ou de faible ruissellement: cas fréquent dans les pays tempérés.

La capacité apparente d'absorption sur un bassin versant homogène donné correspond à l'ensemble des pertes que subit une averse pendant l'unité de temps, à partir du moment où se produit le ruissellement superficiel. On la mesure généralement en $\mathrm{mm} /$ heure.

\author{
J. RODIER \\ INǴÉNIEUR EN CHEF A ĹLECTRICITÉ DE FRANCE \\ CHEF DU SERVICE HYDROLOGIQUUE \\ DE L'OFFICE DE XA RECHERCHE SCIENTIFIQUF, \\ ET TECHNIQUE OUTRE-MEA
}

A difficulty encountered when preparing a flood lydrograph for a particular storm is that of estimating the infiltration capacity.

A great number of factors are involved when determining the infiltration capacity of permeable land.

An experimental determination of the amonnt of rain after which runoff begins, makes it possible to reduce the problem to one of estmating the infiltration capucuty of a heterogeneous surface and this is much easier.

The Porcher method provides a solid basis from which to extrapolate infiltration capacities for heavy precipilation.

This makes the forecasting of exceptional floods a much less random procedure.

The method, which is applicuble to land of medium and very great permeability, should be of much use were there is much vegetational cover or little runoff, conditions which frequently occur in temperate countries. 


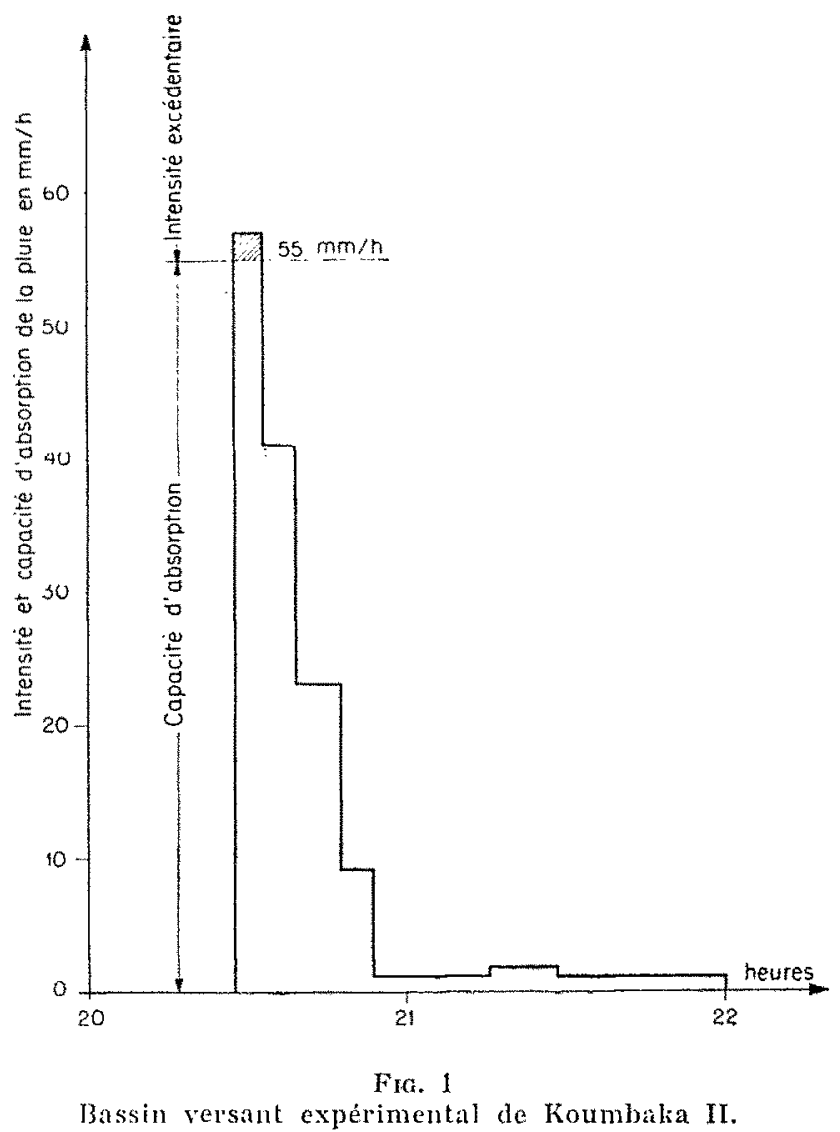

Détermination de la capacité d'absorption. Averse $n^{\circ} 13$.

l'aire représentant le volume de ruissellement tel qu'il a pu être mesuré sur l'hydrogramme correspondant.

On limite souvent cette aire vers le bas par une parallèle à l'axe des temps. Si le ruissellement se prolongeait, il faudrait tracer une courbe de séparation décroissante pour tenir compte de l'augmentation de la saturation avec la duréc de l'averse. L'ordonnée de cette droite de séparation ou l'ordonnée moyenne de la courbe est la capacité apparente moyenne d'absorption. Elle comprend, en fait, des pertes de nature très variée: non seulement des pertes par infiltration, mais encore des pertes par interception, par mise en réserve à la surface du sol et, dans une faible mesure, par évaporation.

Cette notion de capacité d'absorption est particulièrement importante, car la connaissance des valeurs qu*elle peut atteindre pour les précipitations d'ordre décennal permet de calculer le volume de ruissellement et le débit de crue décennale.

Malheureusement, la mise au point du diagramme de variation de la capacité d'absorption moyenne en fonction des diverses caractéristiques de l'averse, sa hauteur par exemple (pour un degré de saturation préalable constant) est beaucoup plus difficile que la détermination du diagramne de distribution.

Dans le cas de terrains assez perméables, le ruissellement n'intéresse qu'une partie du bassin. Le volume de ruissellement est faible par rapport au volume total de précipitations. La construction exposée plus haut, qui n'est valable que pour un bassin homogène, conduit à une valeur de la capacité d'absorption voisine de l'intensité maximale des précipitations. Dans ces conditions, cette caractéristique n'a plus grande signification physique et elle défie toute extrapolation.

Jusqu'à ces derniers temps, on employait, pour le calcul des volumes de ruissellement, la capacité d'absorption de préférence au coefficient de ruissellement, mais la dispersion restait encore Irès grande dans les relations entre la capacité d'absorption et les facteurs physiques dont elle dépend, par exemple la hauteur de précipitation. utile. M. André Bouchardeau a songé alor's à utiliser un diagramme que l'on trace quelquefois au cours des études sur bassins expérimentaux pour déterminer à partir de quelle hauteur de précipitation il $\mathrm{y}$ a ruissellement.

Si, sur un bassin versant situé dans une région où prédominent les tornades, on distingue les pluies ayant donné lieu à ruissellement de celles n'ayant produit aucun résultat, on constate que le ruissellement commence pour une hauteur de précipitation-limite assez bien définie, qui varie en fonction de l'intervalle de temps à l'averse précédente (1). Cette variation est lente, en général, entre la denxième et la soixantième heure.

La figure 2 montre, en coordonnées semi-logarithmiques, comment on peut tracer la courbe de cette précipitation-limite en fonction de l'intervalle.

La dispersion est faible, sauf pour des intervalles de 5 ou 10 jours et plus; ces dernières averses correspondent souvent au début ou à la fin de la saison des pluies, époques pour lesquelles les conditions de ruissellement sont beaucoup plus complexes. O'n trouve également quelques points aberrants pour des ruissellements partiels ou pour des averses dont le hyètogramme est de forme tout à fait anormale. Il existe cependant une limite bien déterminée, indépendante de l'intensité de l'averse, qui résulte du fait que les hyètogrammes des différentes tornades sont de formes très voisines.

(1) Ne sont prises en compte, pour les averses précédentes, que les précipitations supérieures à 5 ou $3 \mathrm{~mm}$. 


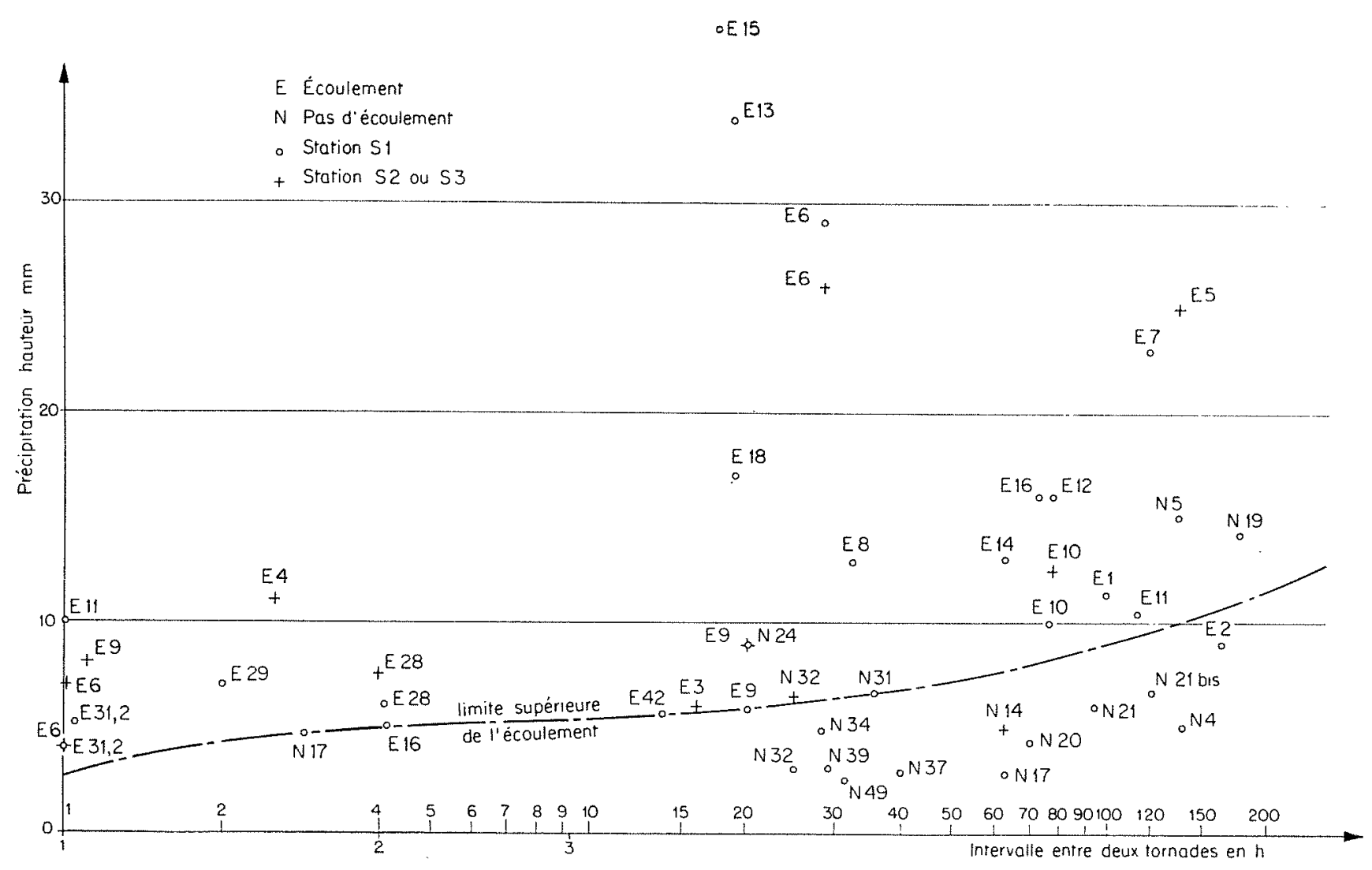

FIg. 2

Recherche de la précipitation limite de ruissellement. Courbe hauteur-intervalle. Campagnes 1956-19:7 (Ouadi Kaoun-Tehad).

La figure 3 montre une série de courbes limites correspondant à des bassins différents, on vérifie que la forme reste la même. La courbe supérieure, NION 2, correspond à un bassin forestier, la courbe inférieure à un bassin comportant des zones imperméables.

A quoi correspond la hauteur de précipitationlimite ainsi définie?

Pour un bassin pas trop hétérogène :

- A la totalité des pertes par interception;

- A la majeure partic des pertes par mise en réserve à la surface du sol;

- A la quantité d'eau nécessaire pour porter les couches superficielles du sol à un degré d'humidification tel qu'il commence à ruisseler, tout au moins pour la majeure partie du bassin;

- A des pertes par évaporation immédiate (négligeables);

- A des pertes par infiltration vers les couches situées au-delà des premiers centimètres de sol, pour les zones les plus perméables.
Les trois premiers postes sont les plus importants, c'est pourcuoi nous appellerons cette hauteur de précipitation-limite: " pluie d'imbibition $\mathrm{P}_{0} \gg$.

On voit que, si l'on néglige des pertes secondaires, l'averse n'a plus $\dot{a}$ subir que des pertes par infiltration lorsqu'elle satisfait aux besoins correspondant à $\mathrm{P}_{0}$.

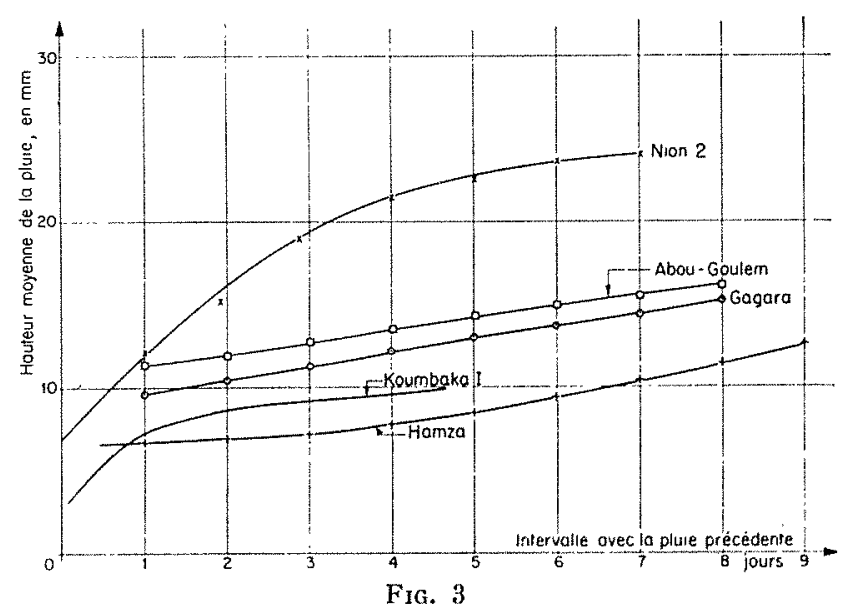

Précipitation limite d'écoulement (ou pluie d'imbibition) pour différents bassins expérimentaux. 
On peut résumer le processus de l'écoulement par le schéma ci-après.

(1) Peut, pour de très faibles intensités. etre perdue par Evaporation directe. (2) Ajouter pertes secondaires par évaporation directe et infiltration.

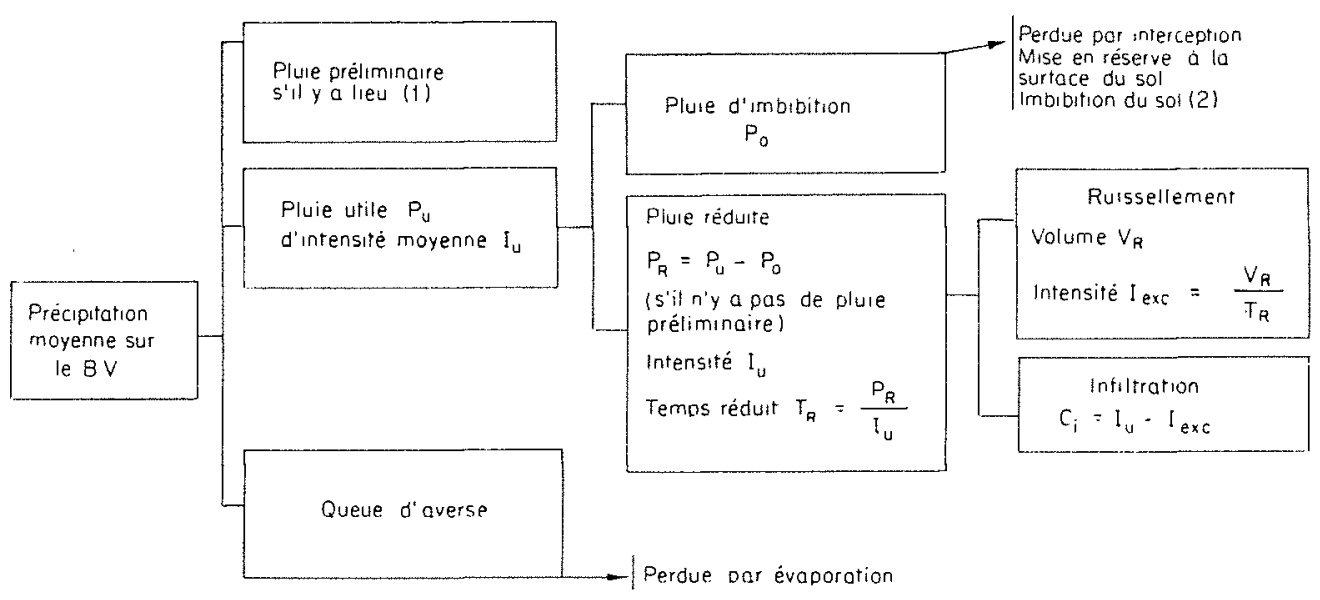

Ce schéma est un peu simplifié, mais il n'est guère possible de procéder à des études d'hydrologie analytique sans simplification de ce genre.

Il montre qu'il est possible de remplacer la détermination de la capacité d'absorption par celui de la capacité d'infiltration en liaison directe avec les propriétés physiques du sol du bassin.

Les opérations nécessaires sont alors les suivantes :

-_. Si la pluie préliminaire est très longue et de très f'aible intensité, la supprimer;

- Amputer ensuite du hyètogramme les premières fractions jusqu'à ce que la hauteur de précipitation ainsi déduite soit égale à $\mathrm{P}_{0}$, pluie d'imbibition;

- Sur le reste du hyètogramme, déterminer la capacité d'infiltration exactement comme

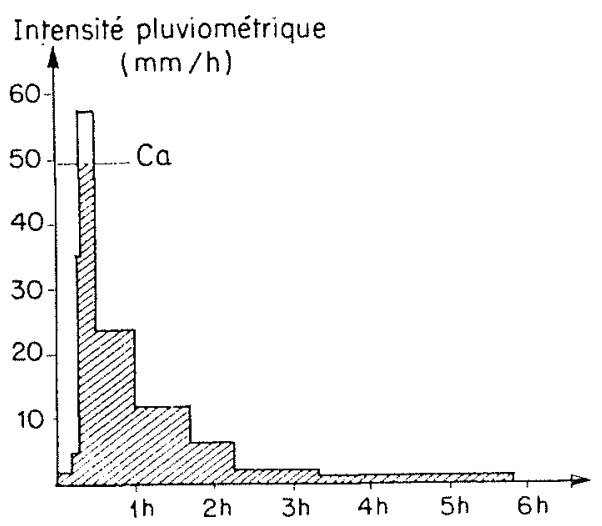

dans lia construction classique; on détermine la capacité d'absorption à partir du volume de ruissellement.

On voit, d'après le graphique 4, combien la différence peut être importante entre les résultats donnés par les deux méthodes. Cette différence est d'autant plus faible que les sols sont plus imperméables et que $P_{0}$ est plus petit. C'est pourquoi, pour les bassins imperméables, à forte pente et à faible couverture végétale, il n'y a aucun inconvénient à conserver le schéma classique et à calculer la capacité d'absorption.

Pour les terrains perméables, au contraire, le calcul de la capacité d'infiltration permet de meilleures corrélations avec les divers facteurs de l'écoulement et il permet également des confrontations avec des mesures directes de la perméabilité.

De telles confrontations ne sont pas simples,

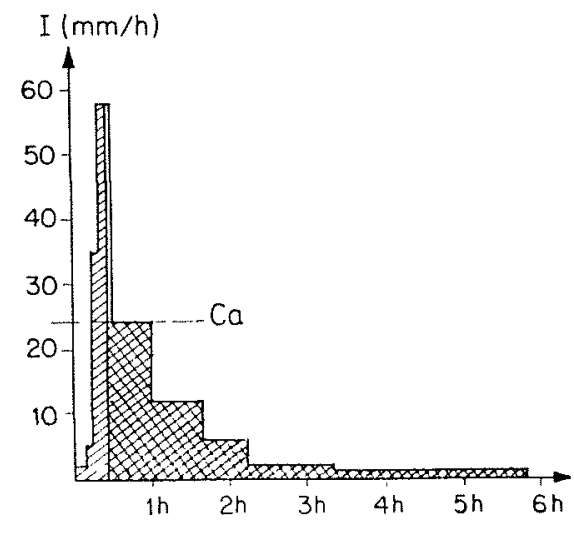

Détermination de la capacité d'absorption apparente.

Méthode classique

$P_{\text {ex.. }}: 1.4 \mathrm{~mm}$,

$C_{a}: 49,5 \mathrm{~mm} / \mathrm{h}$.
Méthode de la «pluie limite» (ou d'imbibition)

Pluie limite $P_{\circ}: 11 \mathrm{~mm}$,

Pluie excédentaire $P_{\text {exc. }}: 1,4 \mathrm{~mm}$,

Capacité d'absorption $\mathrm{C}_{a}: 24 \mathrm{~mm} / \mathrm{h}$. 
car un bassin naturel est rarement homogène (1). Si un bassin expérimental est homogène, il y a souvent intérêt à l'abandonner, car il ne correspond pas alors à des conditions naturelles. Or, l'hétérogénéité a une influence beaucoup plus marquée en terrains perméables qu'en terrains peu perméables.

Le premier effet de l'hétérogénéité d'un bassin est assez surprenant : si l'on considère une série de précipitations d'assez courte durée ef d'intensité croissante, la capacité d'infiltration croît avec l'intensité de l'averse au lieu de décrô̂tre sous l'effet d'une meilleure saturation du sol, comme on pourrait s'y attendre.

L'explication est facile cependant : divisons le bassin en parcelles de superficie $A_{i}$ classées par perméabilité croissante $P_{i}$.

Soit $Q_{n}$ le débit de ruissellement et I l'intensité de la pluie supposée constante dans le temps, et de durée suffisante pour que l'infiltration ait atteint le régime d'équilibre :

$$
\mathrm{Q}_{\mathrm{n}}=\sum_{1}^{p}\left(\mathrm{I}-\mathrm{P}_{i}\right) \mathrm{A}_{i}
$$

Senles figurent dans cette relation les premières parcelles pour lesquelles $P_{i}<I$.

Soit $I_{\text {exe }}$ l'intensité excédentaire (pluie nette),

A la superficie totale du bassin :

$$
\mathrm{I}_{\mathrm{exc}}=\frac{\mathrm{Q}_{\mathrm{r}}}{\mathrm{A}}=\sum_{1}^{p}(\mathrm{I}-\mathrm{P} i) \frac{\mathrm{A}_{i}}{\mathrm{~A}}
$$

La capacité d'infiltration de l'ensemble devient alors :

$$
\begin{aligned}
\mathrm{C}_{i} & =\mathrm{I}-\mathrm{I}_{\mathrm{exc}}=\mathrm{I}-\sum_{\mathrm{I}}^{p}\left(\mathrm{I}-\mathrm{P}_{i}\right) \frac{\dot{A}_{i}}{\mathrm{~A}} \\
& =\mathrm{I}\left(1-\sum_{1}^{p} \frac{\mathrm{A}_{i}}{\mathrm{~A}}\right)+\sum_{1}^{p} \frac{\mathrm{P}_{i} \mathrm{~A}_{i}}{\mathrm{~A}}
\end{aligned}
$$

- Pour des valeurs de $I$ inférieures à la plus faible perméabilité $P_{1}$ :

$$
\mathrm{C}_{i}=\mathrm{I}
$$

- Pour I légèrement supérieure à $P_{1}$ :

$$
\sum_{1}^{p}\left(I-P_{i}\right) \frac{A_{i}}{A} \# 0
$$

donc $C_{i}$ est très voisin de $P_{1}$.

- Pour $I \geqslant P_{n}$ correspondant à la perméabilité maximum :

$$
\begin{aligned}
\sum_{1}^{n} \frac{A_{i}}{A} & =1 \\
C_{\mathrm{i}} & =\sum_{1}^{n} \frac{P_{i} A_{i}}{A}
\end{aligned}
$$

(1) Sauf le cas des plateanx Batékés, par exemple.
$\mathrm{C}_{i}$ est la moyrenne pondérée des perméabilités, indépendante de $\mathrm{I}$ et supérieure à $\mathrm{P}_{1}$.

Donc $\mathcal{C}_{i}$ croît d'une valeur voisine de $P_{1}$ à $\sum_{1}^{n} \frac{P_{i} A_{i}}{A}$

quand I croît de $P_{i} \grave{a} P_{n}$.

Au-delà, $C_{i}$ reste constant quand I croít.

Si la durée de l'averse se prolonge, le sol se sature de plus en plus et $\mathrm{C}_{i}$ diminue. On retrouve le schéma classique.

Mais, pour des bassins perméables ou très perméables, après déduction de la précipitation d'imbibition $P_{0}$, il est assez fréquent que $I$ ne puisse pas atteindre $P_{n}$, c'est-à-dire que le bassin ne ruisselle jamais en totalité.

Pour les valeurs de I faibles, on retrouve simplement I quand on cherche $C_{i}$. C'est ainsi le cas pour les denses couvertures forestières, pour lesquelles la connaissance de l'intensité maximale est sans intérêt.

Nous avons raisonné sur des intensités croissantes. On pourrait penser que l'on retrouverail des phénomènes analogues, quoique moins nets pour une valeur constante des intensités de toutes les averses observées et une durée croissante. Si la valeur constante considérée est assez élevée, il a été constaté des phénomènes analogues, quoique la véritable saturation intervienne rapidement dans les parties les moins perméables. Il faut pour cela que le régime pluviométrique admette des pluies assez fortes, prolongées pendant plusieurs heures, ce qui n'est pas le cas des tornades toujours assez brèves.

En terrains perméables, les mesures directes d'infiltration par la méthode Muntz peuvent donner une assez bonne idée de la perméabilité des diférentes fractions du bassin. Supposons ces perméabilités déterminées en $\mathrm{mm}$ /heure; l'équation (3) montre que l'on peut déterminer par une construction simple la capacité d'infiltration pour d'assez courtes précipitations d'intensité constante dans le temps (fig. 5).

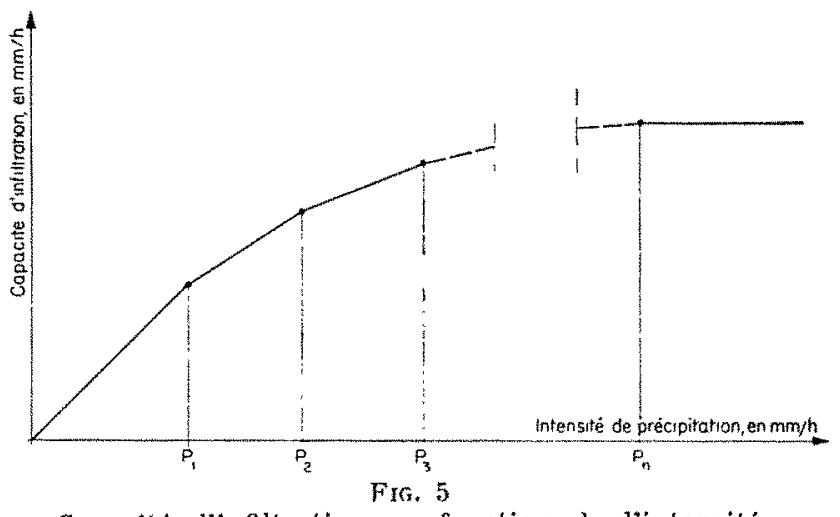

Capacité d'infiltration en fonction de l'intensité supposée constante pendant toute la durée de l'averse. 
Pour I très faible, $\mathrm{C}_{i}=\mathrm{I}$.

Pour I supérieure à $\mathrm{P}_{1}$, mais inférieure à $\mathrm{P}_{2}, \mathrm{C}_{i}$ est donné par la droite:

$$
C_{i}=I\left(1-\frac{A_{1}}{A}\right)+\frac{P_{1} A_{1}}{A}
$$

équation d'une droite moins inclinée sur l'axe des abscisses que la droite $\mathrm{C}_{i}=I$.

Pour I supérieure à $\mathrm{P}_{1}$, mais inférieure à $\mathrm{P}_{2}, \mathrm{C}_{i}$ est donné par la droite :

$$
C_{i}=I\left(1-\frac{A_{1}+A_{2}}{A}\right)+\frac{P_{1} A_{1}+P_{2} A_{2}}{A}
$$

dont la pente est plus faible que celle de la droite précédente.

$$
\text { Pour } A_{i}=A_{n}, \frac{A_{1}+\ldots A_{n}}{A}=1
$$

$C_{i}$ reste constant.

Connaissant $\mathrm{I}, \mathrm{P}_{1} \ldots \mathrm{P}_{n}$ et $\mathrm{A}_{1} \ldots \mathrm{A}_{w}$, il est facile de déterminer $\mathrm{C}_{i}$.

En réalité, I n'est pas constante pendant la durée de la pluie utile, et celle-ci n'est pas la même pour toutes les averses.

Mais, dans le cas de tornade, la durée de la pluie utile est courte et reste du même ordre de grandeur, $15 \mathrm{mn}$ à 1 heure; les intensités restent fortes pendant cette période; on ne commet pas une grande erreur en remplacant cette pluie utile par une averse d'intensité constante, égale à la moyenne des intensités, à condition de déterminer avec beaucoup de soin le début de la traîne à éliminer, ce qui est parfois délicat. On conserve alors le même schéma de calcul que plus haut.

En pratique, on trace le schéma du diagramme 5 d'après les mesures faites à l'infiltrometre Muntz, et on reporte sur le diagramme les points expérimentaux déduits du bilan du bassin, par la méthode décrite au début de cet exposé.

Si ces points sont assez voisins de la ligne brisée du diagramme, on peut s'inspirer de ce diagramme pour extrapoler au-delà des plus fortes intensités directement observées.

On voit immédiatement que ceci ne serait pas valable pour le cas où le terrain commencerait à se saturer, mais ceci n'est pas à craindre sur les sols perméables, pour lesquels, nous l'avons déjà dit, I n'atteindrait pas $\mathbf{P}_{n}$.

On peut alors se demander s'il est bien utile d'observer pluies et averses sur un bassin et s'il ne suffirait pas d'étudier les perméabilités à l'infiltromètre Muntz par exemple.

Nous estimons que, dans l'état actuel de nos connaissances, ce serait fort imprudent. L'action de la couche d'eau qui, dans l'infiltromètre Muntz, surmonte les parties superficielles du sol, est différente de l'action dynamique de la pluie et elle ne donne pas les mêmes résultats dans de nombreux cas. Il arrive que la perméabilité déterminée par cel appareil soit systématiquement trop forte. Il est donc bon de confronter d'abord les résultats ainsi obtenus avec ceux provenant $d u$ bilan sur le bassin, pour vérifier si ces essais sont bien représentatifs, surtout pour les intensités assez fortes. D'autre part, certaines particularités du réseau hydrographique peuvent perturber le processus simple que nous avons décrif. Il faut considérer notre ligne brisée uniquement comme un guide pour l'extrapolation.

L'utilisation de ces méthodes ne doit pas rester limitée aux régions tropicales. Nous pensons que la notion de pluie d'imbibition peut parfaitement ètre utilisée dans des régions tempérées où les pluies ne sont pas très violentes et où la couverture végétale est suffisante pour limiter le ruissellement à très peu de chose, car ces méthodes ont précisément été mises au point pour de faibles ruissellements.

Dans ce cas également, la perméabilité du bassin est hétérogène : seules ruissellent pour les pluies courantes, les routes, sentiers, cours et toitures.

En forêt également, les précipitations n'arrivent au sol qu'après un premier seuil do précipitations; un second seuil est à franchir pour obtenir une sorte d'écoulement que, pour simplifier, on peut appeler ruissellement. On peut définir une pluie d'imbibition qui englobe ces deux seuils.

Les phénomènes seront cependant un peu différents du cas précédent; une fois la limite d'imbibition satisfaite, la forêt opérera une certaine régularisation sur la dentelle du diagramme d'intensité. C'est la raison pour laquelle, en forêt tropicale, nous n'accordons qu'une faible importance aux intensités; seule compte la hauteur de pluie utile. 


\section{I S C S I I N}

Président : M. Liğouzat

M. le Président remercie beaucoup M. Ronner.

M. Remenieras insiste sur l'intérêt de la notion d'imbibition présentée par M. Rodren : elle s'apparente aux conceptions d'un spécialiste russe, M. AlexNorf, qui ne prend en compte que les «pertes initiales $\gg$ de l'averse - ce qui n'est évidemment qu'une apploximation moins satisfaisante que celle proposée par MM. Bouchardeau et Ropier.

M. Ronrsr estime que cette simplification est assez hardice, car l'intérết de cette notion d'imbibition est d'éliminer l'interception, de ramener toutes les averses aux mêmes conditions initiales, et surtout de permettre l'étude de la véritable infiltration.

M. Remenienas ajoute, à la décharge de M. Alexmofr, que l'étude visée ne porte que sur de très grandes crues.

M. Rodrer précise que M. Alexnoff ètudie, peut-être, des pluies de très fortes intensités tes terrains de perméabilité relativement faible.

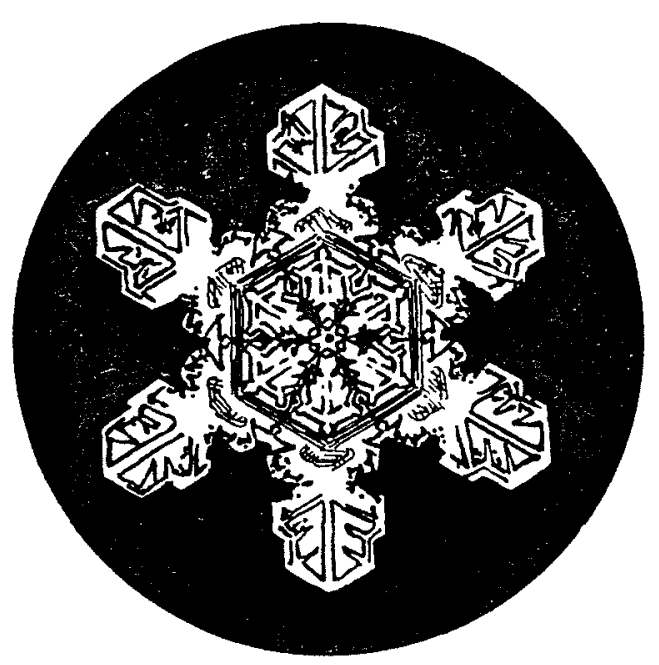

